\title{
Use of a Rotated Riemann Solver for the Two-Dimensional Euler Equations
}

\author{
David W. Levy, Kenneth G. Powell, and Bram van Leer \\ Department of Aerospace Engineering, University of Michigan, Ann Arbor, Michigan $4810 \mathrm{y}$
}

Received March 4, 1991; revised September 24, 1992

\begin{abstract}
A scheme for the two-dimensional Euler equations that uses flow parameters to determine the direction for upwind-differencing is described. This approach respects the multi-dimensional nature of the equations and reduces the grid-dependence of conventional schemes. Several angles are tested as the dominant upwinding direction, including the local flow and velocity-magnitude-gradient angles. Roe's approximate Riemann solver is used to calculate fluxes in the upwind direction, as well as for the flux components normal to the upwinding direction. The approach is first tested for two-dimensional scalar convection, where the scheme is shown to have accuracy comparable to a high-order MUSCL scheme. Solutions of the Euler equations are calculated for a variety of test cases. Substantial improvement in the resolution of shock and shear waves is realized. The approach is promising in that it uses flow solution features, rather than grid features, to determine the orientation for the solution method. (C) 1993 Academic Press. Inc.
\end{abstract}

\section{INTRODUCTION}

When approximating hyperbolic partial-differential equations in one dimension by upwind differencing, finding the upwind direction is straightforward. Characteristic information can only be transmitted forwards or backwards depending on the sign of the corresponding characteristic speed. In two or three dimensions the choice is more difficult. In the finite-volume formulation of the multidimensional Euler equations, neighboring cells are assumed to interact through plane waves propagating normal to the common cell face: the solution to the local Riemann problem. Upwind-biased fluxes may then be derived just as for a one-dimensional system of equations. The problem with this "grid-aligned" approach is that it can misrepresent the physical features of the flow, unless they happen to lie along the grid direction. This becomes particularly evident when computing oblique waves, which tend to become excessively smeared.

Consider, for example, a pure, steady shear wave oblique to the grid, as shown in Fig. 1. The component velocity normal to the wave is zero on both sides of the wave-only the tangential component changes. But in the grid-aligned frame, the component normal to the cell face will change. The solution to the Riemann problem in the grid-aligned frame will thus include a shock wave or an expansion wave, depending on the sign of the velocity change. This incorrect interpretation of the discrete solution in terms of moving acoustic waves causes the shear wave to spread.

The present research examines the use of a "rotated Riemann solver," in which the upwinding angle is determined not by the grid orientation but by physical features of the flow problem. The goal is to lessen the dependence of the solution on grid orientation by cxploiting the multidimensional nature of the equations.

Numerical methods based on wave propagation may be categorized in the following manner:

1. Grid-aligned methods, in which the fluxes are calculated in a reference frame aligned with the grid and cell-centered data are used directly to calculate these. These methods have found widespread use in practice $[1,2]$.

2. Rotation methods, in which fluxes are calculated in coordinates other than a grid-aligned system, but the same cell-centered data are used to calculate these as in gridaligned methods. The rotation of the coordinate system is dictated by physical flow features [3-6].

3. Rotation/interpolation methods, in which fluxes are calculated in coordinates other than a grid-aligned system, and an interpolation process is used to estimate data at desired locations in the flow field, needed for the calculation. The rotation of the coordinate system and the interpolation locations are dictated by physical flow features [7-9].

4. Truly multi-dimensional convection schemes, in which as little "one-dimensional thinking" as possible is used. In these methods it is possible for different rotational frames to be used for different variables [10-12].

Most current schemes fall into the first category, because it is one of the simplest and most economical implementa- 


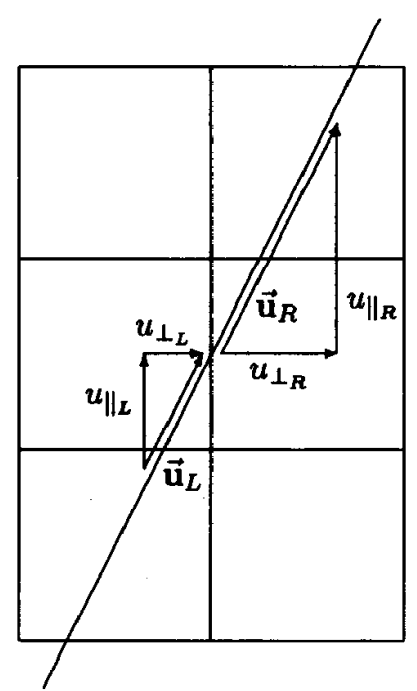

FIG. 1. Pure shear wave oblique to grid.

tions possible. The typical drawback has been discussed above.

Work on rotation-type schemes includes the solution of the transonic potential equation in intrinsic coordinates by Jameson [3]. For the Euler equations, the $\lambda$-scheme developed by Moretti [13] uses certain characteristic directions, while the related QAZ1D method of Verhoff et al. [4] and the method of Goorjian $[5,6]$ use intrinsic coordinates. The present work began as an extension of the QAZ1D method. The wave-decomposition scheme of Rumsey et al. [14] uses a fundamentally different Riemann solver for the flux calculation.

Examples of rotation/interpolation methods applied to scalar convection are the "skew upwind scheme" due to Raithby [7], and the " $N$-scheme" of Sidilkover [8]. Davis [9] has implemented a scheme for the Euler equations with upwind differencing in the frame normal to the shock wave. A finite-volume scheme similar to the present work is given by Dadone and Grossman [15].

A method that belongs to the class of truly multi-dimensional schemes is the characteristic interpolation scheme of Hirsch and Lacor [10], where certain characteristic variables are interpolated along associated dominant directions. Other current efforts to develop truly multi-dimensional schemes were made by Powell and Van Leer [11] and by Struijs and Deconinck [12]. They consider cellvertex schemes with a downwind-weighted residual distribution. The diagonalization of Hirsch [16] (same as in [10]) for the multi-dimensional Euler equations is used for wave decomposition, and the convection of each characteristic variable is oriented in the proper direction. A similar approach, using a different wave model, is described in Roe [17] and has been implemented by De Palma et al. [18].

A summary of the wave-decomposition schemes, the truly multi-dimensional schemes, and the present scheme is given in Powell et al. [19] and Roe [20]. Comparative results are also presented.

The present method belongs to the class of rotation/interpolation schemes. In all such schemes, the flow is assumed to be dominated by one or more waves; a technique is devised to achieve finite-differencing normal to the wave front(s) because that results in the best resolution of discontinuities. Implicit in this approach (even if the scheme is grid-aligned) are three major solution steps:

1. Choice of the upwind differencing angle;

2. Determination of a "left" and a "right" state at each cell interface, as a function of the upwinding angle;

3. Computation of fluxes at the appropriate angle.

In the rotation and rotation/interpolation schemes listed above, various combinations of upwinding angle and interpolation technique appear. Different upwinding angles are used, and several sources of input data are tried, ranging from grid-aligned states to interpolated values. With the exception of Davis' scheme [9] and the present scheme, these schemes compute the flux in a grid-aligned coordinate system. The attraction of the rotation/interpolation approach is that it allows the study of the effect of using a rotated reference frame, while at the same time standard Riemann solvers are used. The truly multi-dimensional schemes require fundamentally new Riemann solvers, which are not yet mature.

The differences between grid-aligned schemes and the present scheme based on a rotated Riemann solver all trace back to differences in the following steps:

1. In a grid-aligned scheme, the direction of upwind differencing is normal to the cell face. In the present scheme, the upwinding angle can be freely chosen, and, in particular, can be based on features such as flow and pressure-gradient angles.

2. In a first-order grid-aligned scheme, the left and right states are taken to be the average quantities in the cells on either side of the cell face. In the present scheme, the left and right state vectors depend continuously on the upwinding direction. The first-order scheme uses interpolation between cell centers to obtain these states.

3. In a grid-aligned scheme, the flux normal to the upwinding direction does not contribute to the flux through the cell face, so it is not calculated. In the present scheme, the fluxes at the chosen angle, as well as in the direction normal to it, must be calculated. The flux normal to the cell face is computed by projecting these two flux components onto the coordinate frame aligned with the cell face.

Before discussing the formulation for the Euler equations, these considerations are examined in the context of the twodimensional scalar convection equation (Section 2). It is then possible to analyze several aspects of the scheme; 
including monotonicity. The scheme is then extended to the two-dimensional Euler equations (Section 3). Various upwinding angles are tested, all using the approximate Riemann solver due to Roe [21]. The lessons learned from the monotonicity analysis of the scalar equation are then applied to the Euler equations. Results for three different flow problems are shown (Section 4). Conclusions and recommendations are presented in Section 5.

\section{SCALAR CONVECTION}

In the development of schemes to model equations that describe convection, it is useful to apply these schemes first to the simple convection equation. In two dimensions, the convection equation is

$$
u_{t}+a u_{x}+b u_{y}=0 .
$$

In general, the coefficients $a$ and $b$ may be functions of $x, y$, or, in the nonlinear case, $u$.

Formulation of the Grid-Aligned Scheme. A first-order accurate, grid-aligned upwind method is formulated by calculating fluxes based on the upwind, cell-centered state values. For example (if $a$ is constant),

$$
F_{i-1 / 2, j}= \begin{cases}a u_{i-1, j}, & a>0 \\ a u_{i, j}, & a<0 .\end{cases}
$$

A better approximation method for the fluxes (although still grid-aligned) is the MUSCL approach, due to Van Leer $[22,2]$. For example, with $a>0$, the flux in Eq. (2) can be computed with

$$
u_{i-1 / 2, j}=u_{i-1, j}+\left\{\frac{s}{4}\left[(1-\kappa s) \Delta_{-}^{i}+(1+\kappa s) \Delta_{+}^{i}\right]\right\}_{i-1, j} ;
$$

the forward and backward difference operators are defined by, respectively,

$$
\left(\Delta_{+}^{i}\right)_{i, j}=u_{i+1, j}-u_{i, j}, \quad\left(\Delta_{-}^{i}\right)_{i, j}=u_{i, j}-u_{i-1, j} .
$$

With $\kappa=\frac{1}{3}$, this corresponds to parabolic interpolation with the stencil centered on $(i-1, j)$. Also included in Eq. (3) is the limiter function $s=s\left(\Delta_{+}, \Delta_{-}\right)$, which is used to prevent overshoots and undershoots near discontinuities. The differentiable limiter due to Van Albada is used:

$$
s\left(\Delta_{+}, \Delta_{-}\right)=\frac{2 \Delta_{+} \Delta_{-}+\varepsilon}{\left(\Delta_{+}\right)^{2}+\left(\Delta_{-}\right)^{2}+\varepsilon} .
$$

The parameter $\varepsilon$ is used to prevent division by zero in regions of uniform flow, where the differences are very small. The value $\varepsilon=1 \times 10^{-10}$ is typically used for double precision calculations.

Formulation of the Characteristic Interpolation Scheme. When the requirement of using grid geometry in the determination of cell face states is relaxed, it becomes apparent that a large class of schemes can be designed in which the cell-face state values are traced back to some point within the domain of dependence for that face. The first step in the specification of the cell-face state is to select a direction for upwind-differencing; the convection angle is a logical choicc. The other important direction to consider is that of the solution gradient. However, in the linear case, the steady convection and solution gradient angles are orthogonal, so these define the same orthogonal frame. In the scalar case, the convection angle defines the characteristic direction, so this method is referred to as "characteristic interpolation."

Once the upwinding angle has been specified, there is still a large range of possibilities for interpolation locations. To simplify the interpolation process, the state locations are chosen to be on lines connecting adjacent cell centers. For example, when $a$ and $b$ are both constants and $0.5 \leqslant b / a \leqslant 2.0$, the interpolation locations for all four faces of a cell are shown in Fig. 2. There are more possibilities, however, in the case of arbitrary convection speeds. Then, a complete template of cells surrounding the cell face of interest must be considered. Linear interpolation of states between cell centers is used,

$$
u^{*}=(1-w) u_{1}+w u_{2},
$$

where $u^{*}$ is the interpolated state, $w$ is the linear interpolation weight, and $u_{1}$ and $u_{2}$ are the two states located at the cell centers which straddle the characteristic line. For cases where $a$ or $b$ are zero, the linear interpolation gives the cell center state and the characteristic interpolation scheme reduces to the grid-aligned scheme.

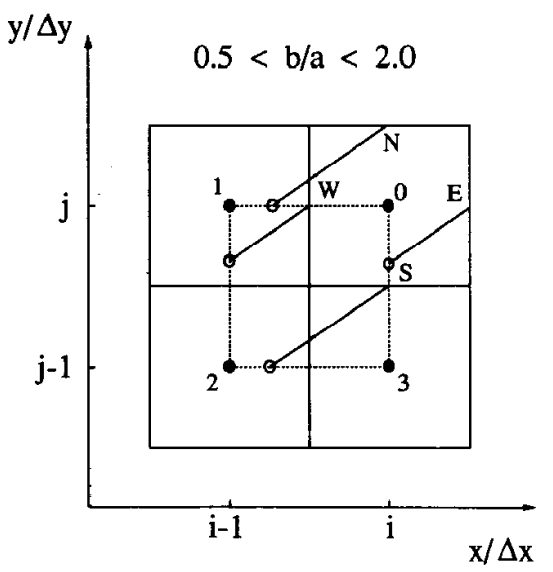

FIG. 2. Locations for interpolation of state quantities. 
The interpolated state values are used as approximations for the cell face states for the flux computation, e.g.,

$$
F_{i-1 / 2, j}=a u_{i-1 / 2, j}^{*}
$$

Analysis of the Characteristic Interpolation Scheme. For the case in which $0.5 \leqslant b / a \leqslant 2$, shown in Fig. 2, the forward Euler update for cell 0 is then dependent only on cells $0-3$ and may be written

$$
\begin{aligned}
u_{0}^{n+1}= & u_{0}^{n}-\frac{\Delta t}{h}\left[\frac{1}{2}(a+b) u_{0}^{n}+\frac{1}{2}(b-a) u_{1}^{n}\right. \\
& \left.+\frac{1}{2}(a-b) u_{3}^{n}-\frac{1}{2}(a+b) u_{2}^{n}\right] .
\end{aligned}
$$

This is equivalent to a cell vertex scheme, in which the residual for the imaginary cell 0123 , centered at vertex $\left(i-\frac{1}{2}, j-\frac{1}{2}\right)$, is calculated using the trapezoidal rule for the fluxes. The residual is then assigned fully to the downwind node, node 0 . The scheme will be second-order accurate in space, but will not be monotone, as shown below.

This level of accuracy is perhaps unexpected, because the characteristic upwinding scheme, from a geometric perspective, would appear to be only first-order accurate. Data used for the face fluxes are taken some distance away from the face itself. However, since the solution of the equation is constant along characteristic lines, the first-order error in the extrapolation to the cell face disappears in the steady state.

The property of positivity can be examined by writing the scheme in the form

$$
u_{0}^{n+1}=\sum_{k=0}^{N} c_{k} u_{k}^{n}
$$

where the sum is over all cells which contribute to the residual. A scheme will be monotone if none of the coefficients $c_{k}$ is negative (see Godunov [23]). Rewriting Eq. (8) in this form reveals:

$$
\begin{aligned}
& c_{0}=1-\frac{1}{2}\left(v_{a}+v_{b}\right), \quad c_{1}=\frac{1}{2}\left(v_{a}-v_{b}\right), \\
& c_{2}=\frac{1}{2}\left(v_{a}+v_{b}\right), \quad c_{3}=\frac{1}{2}\left(v_{b}-v_{a}\right),
\end{aligned}
$$

where $v_{a}=a \Delta t / h$ and $v_{b}=b \Delta t / h$ are the Courant numbers in the $x$ and $y$ directions. It can be seen that $c_{0}>0$ if $v_{a}<1$ and $v_{b}<1$. The coefficient $c_{2}$ is always positive, but $c_{1}$ and $c_{3}$ cannot be positive simultaneously. They can both be zero, when $v_{a}=v_{b}$.

In [8], Sidilkover derives a monotone scheme, termed by him the " $N$-scheme." It can be interpreted in a geometric manner as a restriction on the interpolation procedure used to find the upwind states. Figure 2 shows the characteristic lines for the case $a>b>0$. The flux formulas obtained from characteristic interpolation for the north and south faces are:

$$
\begin{aligned}
& F_{N}=\left[\left(b-\frac{a}{2}\right) u_{0}+\frac{a}{2} u_{1}\right] \\
& \left.F_{S}=\left[\left(b-\frac{a}{2}\right)\right] u_{3}+\frac{a}{2} u_{2}\right] .
\end{aligned}
$$

Sidilkover observed that modifying the weights in these formulas to obtain positivity can be regarded as the result of limiting the angle by which each characteristic deviates from the cell-face normal. If the upwinding angle is restricted to $\pi / 4$ for these two faces, then the fluxes become

$$
\begin{aligned}
& F_{N}=\frac{1}{2} b\left(u_{0}+u_{1}\right) \\
& F_{S}=\frac{1}{2} b\left(u_{3}+u_{2}\right),
\end{aligned}
$$

and the coefficients that result are

$$
c_{0}=1-v_{a}, \quad c_{1}=v_{a}-v_{b}, \quad c_{2}=v_{b}, \quad c_{3}=0 .
$$

Since $a>b>0$, this is a positive scheme. A similar restriction on the interpolation for the vertical faces works for the case $b>a$. The full range of monotone upwinding angles is shown in Fig. 3.

Results and Discussion. The first-order grid-aligned, $\kappa=\frac{1}{3}$ grid-aligned, characteristic interpolation, and limited (restricted) interpolation schemes are tested on two scalar convection problems, both on the domain $0 \leqslant x \leqslant 1$ and $0 \leqslant y \leqslant 1$. Both problems may be described as "circular convection," where the convection speeds are defined by $a=a(y)=y$ and $b=b(x)=\frac{1}{2}-x$. Particles should follow

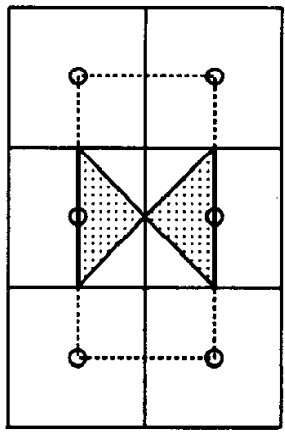

Vertical Faces

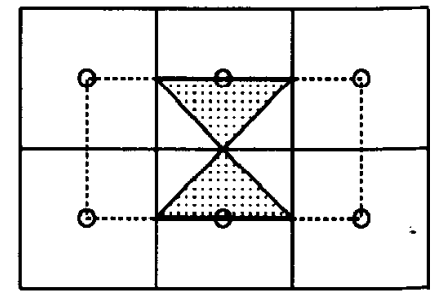

Horizontal Faces
FIG. 3. Limited domain of dependence for the monotone characteristic interpolation scheme. 
clockwise circular paths about the location $\left(\frac{1}{2}, 0\right)$. In the first case, the lower inflow boundary is split up as follows:

$$
\begin{array}{rr}
0 \leqslant x<0.125, & u=0 \\
0.125 \leqslant x<0.375, & u=1 \\
0.375 \leqslant x<0.50, & u=0 .
\end{array}
$$

This describes a "tophat" shape, and will test the four schemes' performance at resolving discontinuities. The second case uses a Gaussian inflow specification at the lower left boundary:

$$
u(x, 0)=e^{-75(x-0.25)^{2}} .
$$

This case will result in a smooth solution, providing a better measure of the schemes' overall accuracy. In each case, the left, upper, and right inflow boundaries are initialized to the exact solution.

Using a $64 \times 64$ cell Cartesian grid, steady-state solutions arc computed. Profiles along the lower boundary for the tophat circular convection are shown in Fig. 4. The data shown are cell vertex valucs, obtained by postprocessing the cell-centered results. The profiles for $0 \leqslant x \leqslant 0.5$ show the input conditions, while the profiles for $0.5<x \leqslant 1.0$ show

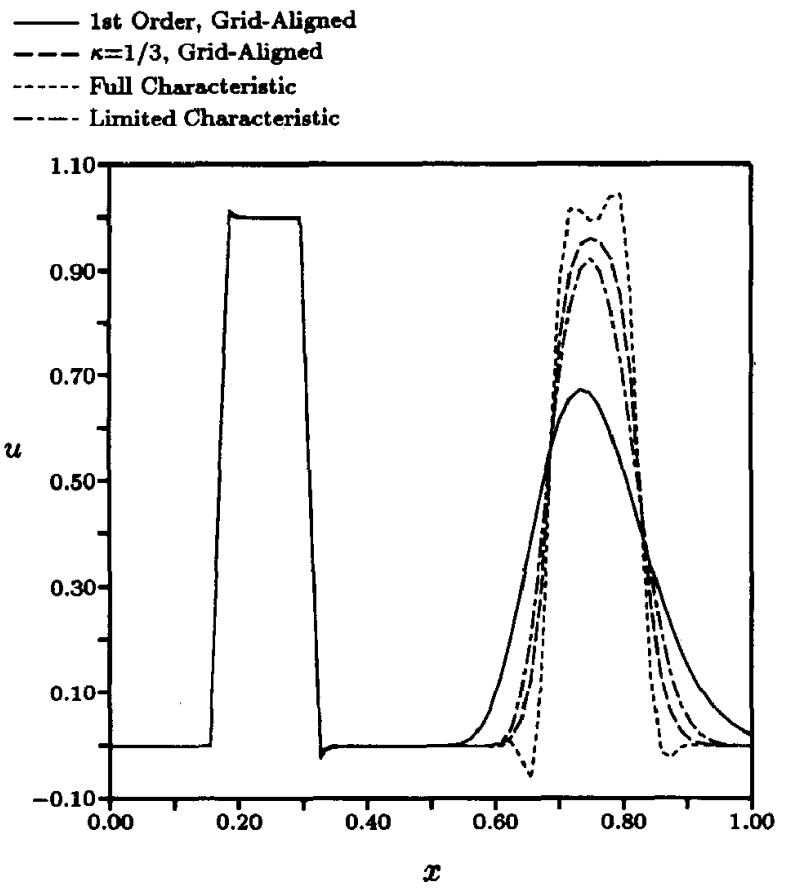

FIG. 4. Tophat circular convection cross section along the lower boundary, computed on a $64 \times 64$ cell grid. the computed solution after convection through the domain.

As expected, the first-order grid-aligned solution is considerably diffused, and the $\kappa=\frac{1}{3}$ grid-aligned scheme gives substantial improvement. The full, or unlimited, characteristic interpolation scheme results in the sharpest of the discontinuities, but at the expense of monotonicity. This is predicted by the positivity analysis above. The angle limiting procedure for the characteristic interpolation scheme works well, giving results comparable to the $\kappa=\frac{1}{3}$ gridaligned scheme. The profiles for the Gaussian case, Fig. 5, show similar trends. In this case, the full characteristic upwinding method preserves the input profile without apparent change.

For this problem, the exact solution is known, so a precise evaluation of the global error is possible. One measure of this is the $L_{2}$ error norm calculated as

$$
L_{2}=\left[\frac{1}{N^{2}} \sum_{i, j=1}^{N}\left(u_{e_{i, j}}-u_{i, j}\right)^{2}\right]^{1 / 2} .
$$

The cell average computed and exact solutions are used to calculate the error. The discrete and exact solutions are calculated for the Gaussian case on $16^{2}, 32^{2}, 64^{2}$, and $96^{2}$ cell Cartesian grids, and the error norms are plotted in Fig. 6. The relative accuracies of the schemes are shown to be the same as indicated by the profile figures. The order of accuracy of the schemes is given by the slopes of the

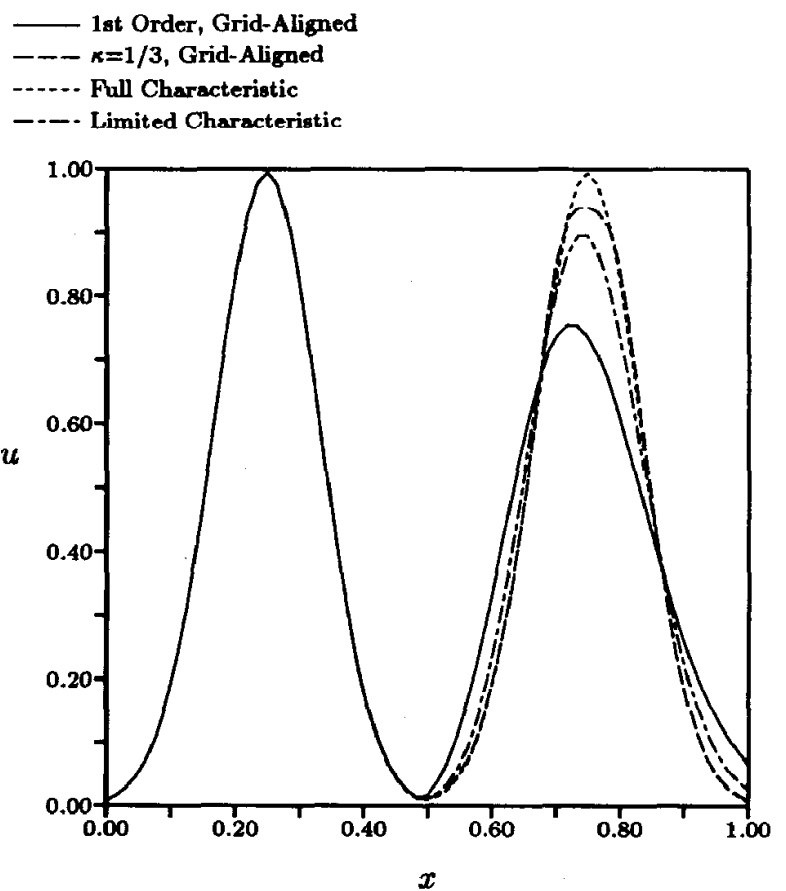

FIG. 5. Gaussian circular convection cross section along the lower boundary, computed on a $64 \times 64$ cell grid. 


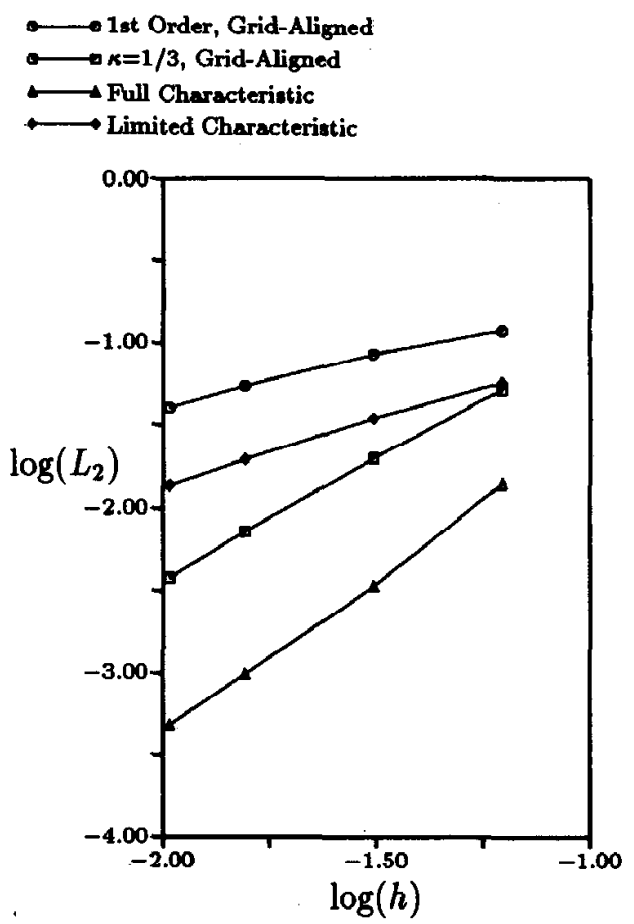

FIG. 6. Error norms for the Gaussian circular convection problem.

$\log \left(L_{2}\right)$ error norms. These are calculated, using linear regression, to be

$$
\begin{array}{ll}
\text { First-order, grid-aligned } & 0.607 \\
\kappa=\frac{1}{3}, \text { grid-aligned } & 1.454 \\
\text { Full characteristic } & 1.879 \\
\text { Limited characteristic } & 0.795
\end{array}
$$

It is seen that the limited characteristic scheme behaves more as the first-order scheme, but with a reduced error coefficient, while the full characteristic scheme mimics the convergence of the $\kappa=\frac{1}{3}$ grid-aligned scheme, again with reduced error.

Overall, the MUSCL scheme produces the best quality results, although the limited characteristic interpolation scheme is a close second. Both of these schemes use interpolation methods similar in complexity, but the characteristic interpolation scheme uses a more compact stencil.

\section{FORMULATION OF THE ROTATED RIEMANN SOLVER METHOD FOR THE EULER EQUATIONS}

In this section, the characteristic interpolation method for scalar convection is adapted to the Euler equations. Although multiple waves may exist locally in the solution-each with its own optimum upwind direction-the present work investigates the resulting improvement when a single, dominant, upwinding angle is used. The conventional Riemann problem, which in grid-aligned methods is solved in a frame normal to the face of a computational cell, is now solved in a frame rotated about the cell face midpoint. This class of scheme will be called "rotated Riemann solvers." In this approach, it is possible to isolate the effects of differing reference frames. In addition, standard flux formulae may be used, which have been in use for some time. The truly multi-dimensional schemes referred to earlier are not as mature and are not as modular in nature.

In formulating the rotated-Riemann-solver method, the major points considered are:

1. Choice of upwind angle;

2. Determination of a left and a right state at each cell interface, as a function of the upwind angle;

3. Computation of fluxes at an appropriate angle.

The performances of the grid-aligned and rotated-Riemannsolver schemes are then compared for channel flows and flows about airfoils in Section 4.

Angles for Upwind Differencing. Since there is more than one characteristic direction for the multi-dimensional Euler equations, a choice must be made. The following choices are considered:

1. Flow angle;

2. Pressure-gradient direction;

3. Velocity-magnitude-gradient direction.

Perhaps the most basic feature in a flow field is the streamline angle, so it is natural to consider it as the dominant direction. This frame is chosen several times in previous work discussed above. The local flow angle at a cell face is computed from the velocities in the neighboring cells $L$ and $R$ :

$$
\theta_{s}=\tan ^{-1}\left(\frac{v_{L}+v_{R}}{u_{L}+u_{R}}\right)
$$

To align the Riemann solver with a shock wave, the pressure-gradient angle can be used. The pressure-gradient field is more difficult to compute than the flow-angle field, but resolution of shock waves should improve if this orientation is used. To compute the pressure-gradient angle, Green's theorem is applied to the scalar pressure, $p$,

$$
\iint_{V} \nabla p d s=\oint_{\partial V} p \hat{\mathbf{n}} d l
$$

where $\hat{\mathbf{n}}$ is the unit normal vector, positive outward. The average pressure-gradient is then

$$
\nabla p_{\text {aug }}=\frac{1}{V} \oint_{\partial V} p \hat{\mathbf{n}} d l
$$


where the integration is in the counterclockwisc dircction. The average pressure gradient at a cell face can then be calculated using an appropriate template of cells surrounding that cell face.

It may also be desirable to align the rotated Riemann solver with pure shear waves, which have no pressure change. In this case, the gradient of the velocity magnitude can be used:

$$
\nabla q=\nabla \sqrt{u^{2}+v^{2}}
$$

This " $q$-gradient" can be calculated in exactly the same manner as the pressure gradient. It has the added benefit of sensing shock waves as well. In the presence of a pure shock wave, pressure-gradient and $q$-gradient upwinding will give identical results.

Interpolation Methods for State Quantities. Once the upwinding direction has been determined, the next step is to determine the state quantities used as inputs to the flux function. An interpolation method is used to approximate the states at a point on the line determined by the upwinding direction and the cell face midpoint. Figure 7 shows a template of six cells surrounding a vertical cell face. As with the characteristic interpolation method used for the scalar convection equation, linear interpolation is used to approximate the states at the point of intersection of the upwinding line and the line connecting the two cell centers that straddle the upwinding line. In the work of Dadone and Grossman [15], the cell average value corresponding to the cell nearest the upwinding line is used. For the Euler equa-
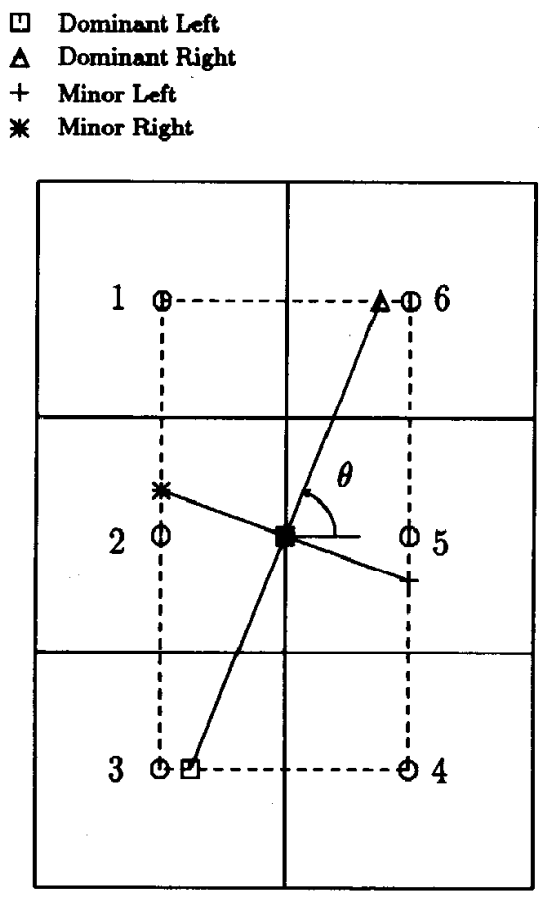

FIG. 7. Interpolation template for the rotated Riemann solver. tions, four locations are required for interpolated states: left and right states for the upwind direction, and left and right states in the normal direction. The states which lie along the upwind line will be called the "dominant" states, while the states which lie on the normal line will be called the "minor" states. A robust searching algorithm, described in detail in [24], is used to identify the locations used for interpolation on nonuniform grids.

With this six-cell template required to calculate the flux through a cell face, a total of nine cells, shown in Fig. 8, will be used to update the cell averaged state values. The cell update template used for the MUSCL scheme is also shown. Whereas the same number of cells are used for each, the rotated-Riemann-solver template is more compact.

Flux Formulation. The generic formula for a flux normal to a cell face is

$$
\mathbf{F}=\mathbf{F}\left(\mathbf{U}_{L}, \mathbf{U}_{R}\right)
$$

in a conventional first-order scheme the left and right input states simply are the average values in the adjacent cells. Convection speeds are then based on velocity components parallel and perpendicular to the cell face; the upwinding direction, i.e., the direction in which the Riemann problem is solved, is normal to the cell face.

When an upwind direction, $\theta$, is chosen that is not normal to the cell face, the flux component in this direction is

$$
\mathbf{F}_{D}=\mathbf{F}_{D}\left(\mathbf{U}_{D_{L}}, \mathbf{U}_{D_{R}}\right)
$$

where the subscript $D$ indicates the input states are found in the upwinding, or dominant, direction; and the flux is formulated along the dominant direction. The dominant direction is chosen, as described above, to be a physically important direction, such as normal to a shock front and not necessarily normal to a cell face. The computation of this flux, therefore, includes two changes with respect to the grid-aligned computation. The velocity components are now in the new reference frame, and the lcft and right states
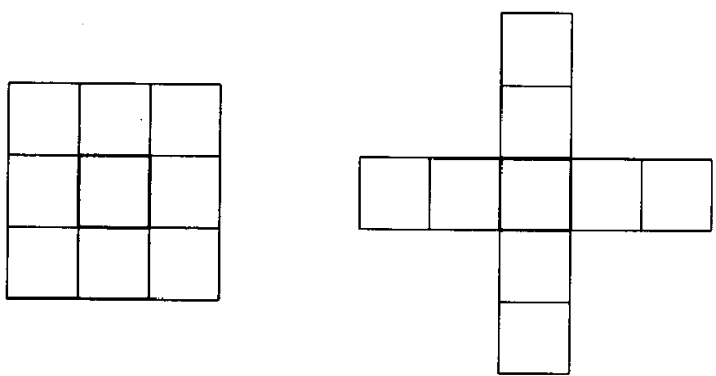

Rotated Riemann Solver

MUSCL Scheme

FIG. 8. Template for cell update for the rotated Riemann solver and the MUSCL scheme. 
are interpolated from the values in the nearest cell centers, as described in the previous section.

Next, a flux normal to this direction, e.g., along a shock front, must also be calculated:

$$
\mathbf{F}_{M}=\mathbf{F}_{M}\left(\mathbf{U}_{M_{L}}, \mathbf{U}_{M_{R}}\right),
$$

where the subscript $M$ indicates that the flux is found in the direction normal to the upwinding angle, or minor direction, and the flux is formulated along the minor direction. It is not a requirement that the minor flux be calculated with the same approximate Riemann solver as the dominant flux. Even simple averaging could suffice. In the final scheme, this would lead to central differencing of the minor flux component. This approach is analogous to that used by Jameson [3] for the potential equation, in which upwind differencing is used for the streamwise derivatives and central differencing is used for the normal derivatives. It is possible to use upwind differencing for both flux components. This would lead to a better approximation of the effect of pressure waves in the minor direction. This, of course, almost doubles the computational effort of calculating the flux through the cell face, relative to a rotated Riemann solver which uses central differencing for the minor flux component. If the dominant direction is the streamline angle, then only the two acoustic waves are required for the computation of the minor flux. If a different direction is used, however, the full Riemann problem must be solved. 'In the work of Goorjian $[5,6]$, which adopts the intrinsic coordinate frame, only the acoustic waves are calculated.

The flux normal to the cell face is constructed by rotating the above fluxes back to the coordinate frame normal to the cell face:

$$
\begin{aligned}
& \mathbf{F}\left(\mathbf{U}_{D_{L}}, \mathbf{U}_{D_{R}}, \mathbf{U}_{M_{L}}, \mathbf{U}_{M_{R}}\right) \\
& \quad=\mathbf{F}_{\mathbf{D}} \cos (\theta-\phi)-\mathbf{F}_{M} \sin (\theta-\phi) .
\end{aligned}
$$

Here, $\theta$ is the upwinding angle and $\phi$ is the angle normal to the cell face. The computation of the upwind biased fluxes in a rotated frame, followed by rotating back to the computational frame, makes a difference only for the nonlinear waves. For passive convection, such as entropy convection, there is no change with respect to grid-aligned fluxes.

In the formulation of the characteristic upwinding scheme for the scalar convection equation, the minor flux component is zero because the convection speed in that direction is zero. It should also be noted that the minor flux component also exists in the conventional, grid-aligned formulation, but does not contribute to the flux through the cell face.

In the earlier work of Davis [9], flux calculation and interpolation are coupled together. The dominant flux is calculated in the rotated frame using unbiased cell average states as in the pure rotation schemes. The minor flux is calculated using certain cell average values, where the specific cells are chosen based on the upwinding angle. It is the flux functions which are interpolated rather than the state quantities.

\section{RESULTS AND DISCUSSION}

In this section, the grid-aligned and rotated-Riemannsolver schemes are used to solve three Euler-flow test geometries and conditions. These are:

1. Fifteen-degree wedge channel flow;

2. Shear wave oblique to the grid;

3. NACA 0012 airfoil.

In the first case, the effects of upwinding direction and minor flux formula are investigated.

Fifteen Degree-Wedge Channel Flow. The geometry for the first test case is a two-dimensional channel with a $15^{\circ}$ wedge on the lower wall. A $15^{\circ}$ expansion corner is also included to study expansion waves and wave interactions. The inflow Mach number equals two. An oblique shock wave is produced by the wedge and is somewhat weakened by the expansion wave. The shock reflects from the upper wall and passes through the rest of the expansion wave. The expansion wave also reflects from the upper wall slightly downstream of the shock-reflection point.

Conventional, analytical calculations predict a postshock Mach number of 1.454. For this Mach number, any turning angle greater than $10.5^{\circ}$ is too large to allow a regular reflection off the upper wall. Thus, a "Mach reflection" results, with subsonic flow behind the Mach stem and a slip surface trailing from the intersection of the incident, normal, and reflected shock waves [25]. In this test case, however, the incident shock wave interacts with the expansion wave. The shock incident on the upper wall may still be too strong to allow a regular reflection; the numerical solutions seem to support this conclusion. The Mach number along the lower wall, after the expansion corner, is predicted to be 1.970 .

The grid used is algebraic, with constant $\Delta x$ and $\Delta y=\left(y_{\max }-y_{\min }\right) / N$, where $N$ is the number of cells in the $y$ direction, as shown in Fig. 9. The first-order grid-aligned upwinding results are shown first in Fig. 10. The shock wave is substantially diffused after reflection, as expected from a first-order-scheme.

Figure 11 shows the result of using the local flow direction as the upwinding direction (streamwise upwinding). The minor fluxes are computed with simple averaging, which leads to central differencing for the minor flux component. Residual smoothing is required to stabilize the scheme. The expansion wave and the reflected shock wave 


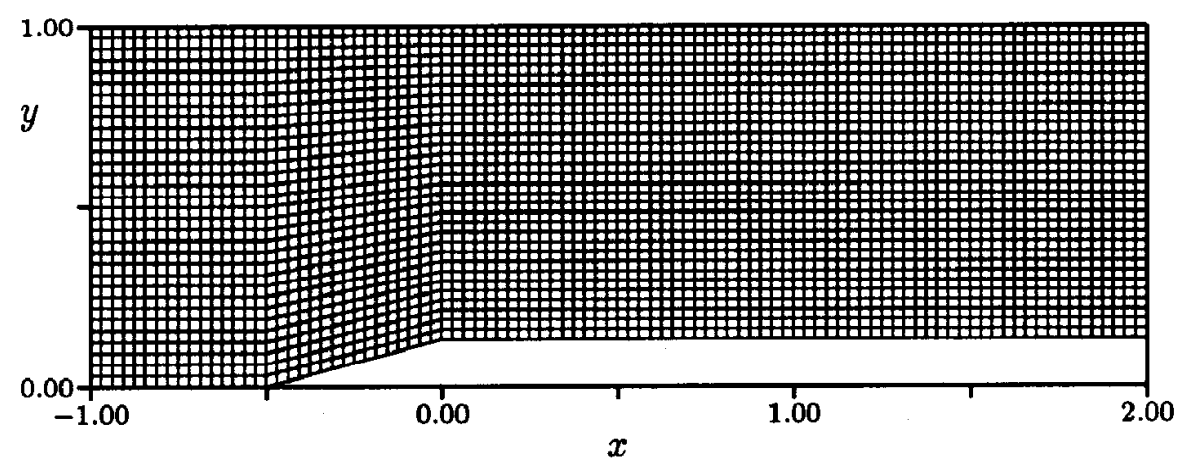

FIG. 9. Algebraic, $96 \times 32$ cell computational grid for the $15^{\circ}$ wedge.

are better resolved. This is especially true of the shock wave reflecting from the lower wall near the exit of the channel. The Mach contours of Fig. 11, and specifically the subsonic Mach levels after the reflected shock $\left(M_{\mathrm{MIN}}=0.9573\right)$, hint at the presence of the analytically predicted Mach reflection. This feature is not present in the first-order grid-aligned upwinding case.

When the flow angle is used for the dominant direction, the only waves traveling in the minor direction are acoustic. It is interesting that central-differencing is stable at all- even without added artificial dissipation.

One reason for the improved resolution and reduced dissipation is that the grid-independent scheme calculates a part of the flux with central-differencing, which is a more accurate approximation $\left(\mathcal{O}(\Delta x)^{2}\right)$ than upwind differencing $(\mathscr{O}(\Delta x))$. In fact, the flux through faces aligned with the local flow direction is entirely composed of the central-differencing component. In the present scheme, the stabilizing effect of using upwind differencing in the dominant direction allows the use of central differencing in the cross direction without artificial viscosity.

The other angle tested is the velocity magnitude, or $q$, gradient angle. The $q$-gradient upwinding, as seen from Fig. 12, shows substantial improvement in the resolution of the shock waves, even over the streamwise upwinding. This should be expected, since now the upwinding direction is normal to shock waves. However, the quality of the solution has degraded in other respects. Overshoots of larger magnitude have appeared in front of the shock, although they are still small. There are noticeable "wiggles" in the contour lines as well, especially in the contours of the expansion fan ahead of the shock reflections.

To avoid noise in regions of uniform flow, a threshold value for the $q$-gradient magnitude of 0.01 of the maximum value in the domain is used. If the $q$-gradient magnitude is greater than the threshold, then that angle is used. Otherwise, the streamline angle is used. Figures 13 and 14 show the upwind directions for the streamwise and $q$ gradient upwinding cases. For these figures, the data have been thinned by a factor two, i.e., directions for every other cell face are plotted. In the streamwise upwinding case, the angles do not deviate much from the horizontal. The alignment normal to the incident and reflected shock waves is quite evident in the $q$-gradient case.

Although the rotated Riemann solvers perform significantly better than the first-order grid-aligned method, the higher-order, MUSCL, grid-aligned scheme still outperforms them all. This is shown in Fig. 15, for $\kappa=\frac{1}{3}$ with Van Albada's limiter. It has comparable shock resolution without overshoots or "wiggles."

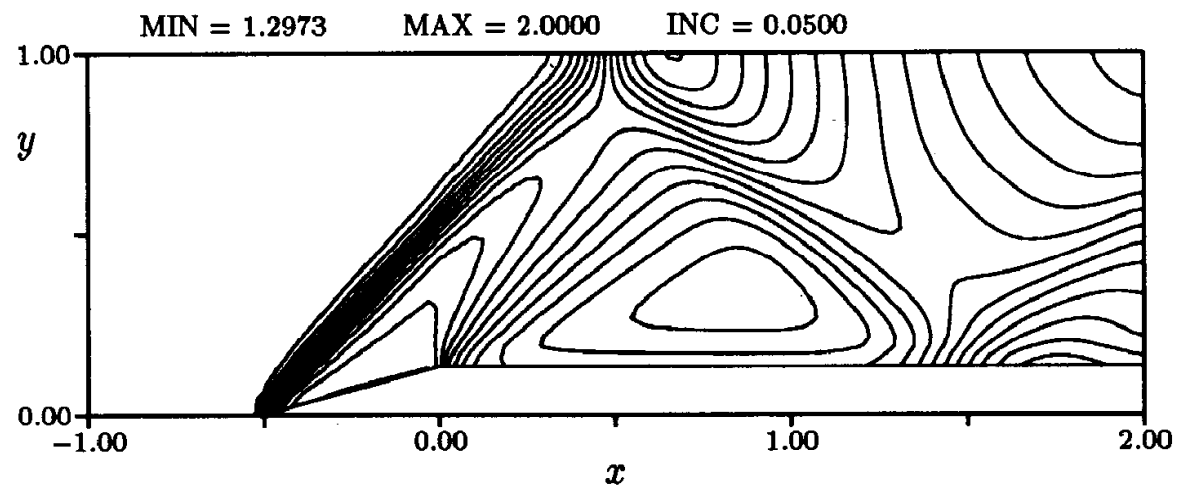

FIG. 10. Mach-number contours for the $15^{\circ}$ wedge flow, first-order grid-aligned upwinding, $96 \times 32$ cell grid. 


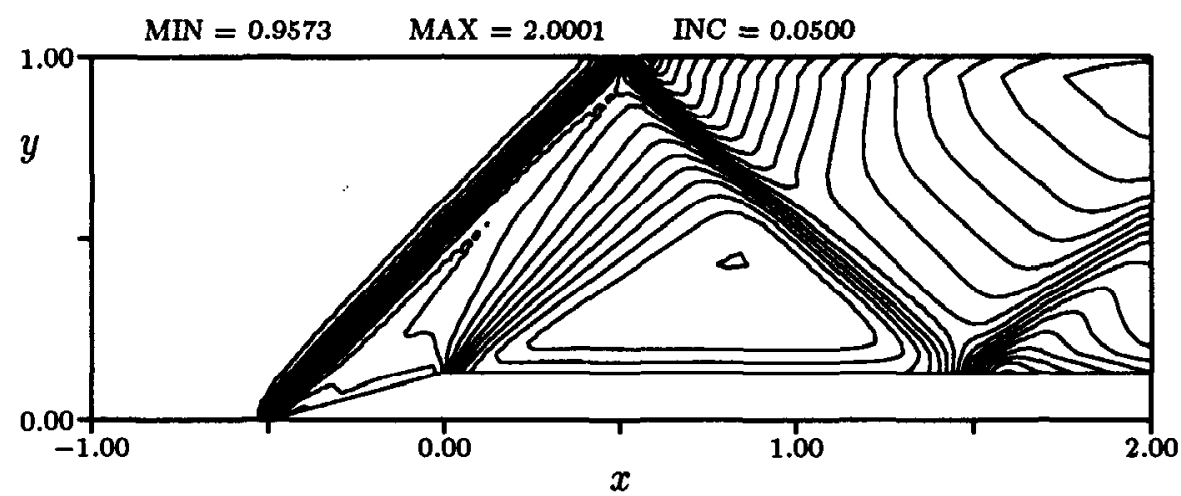

FIG. 11. Mach-number contours for the $15^{\circ}$ wedge flow, first-order streamwise upwinding, central differencing for minor fluxes, $96 \times 32$ cell grid.

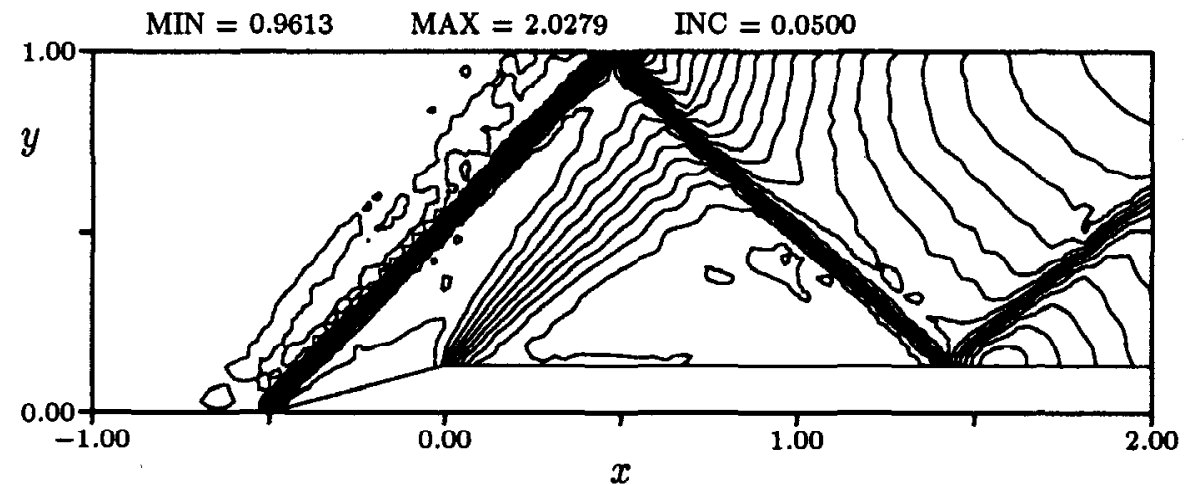

FIG. 12. Mach-number contours for the $15^{\circ}$ wedge flow, first-order $q$-gradient upwinding, central differencing for minor fluxes, $96 \times 32$ cell grid.

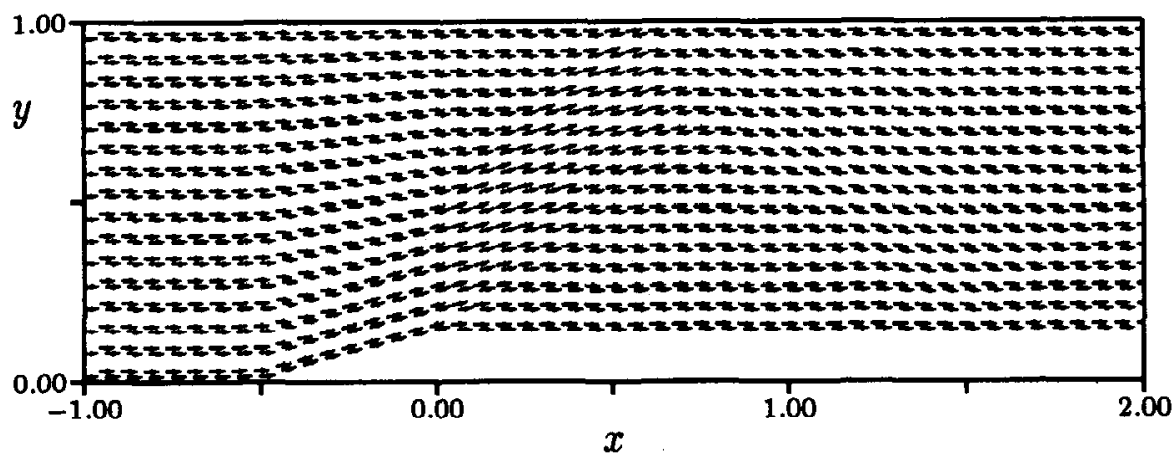

FIG. 13. Upwinding angles for the $15^{\circ}$ wedge flow, streamwise upwinding, $96 \times 32$ cell grid, thinning factor: 2 .

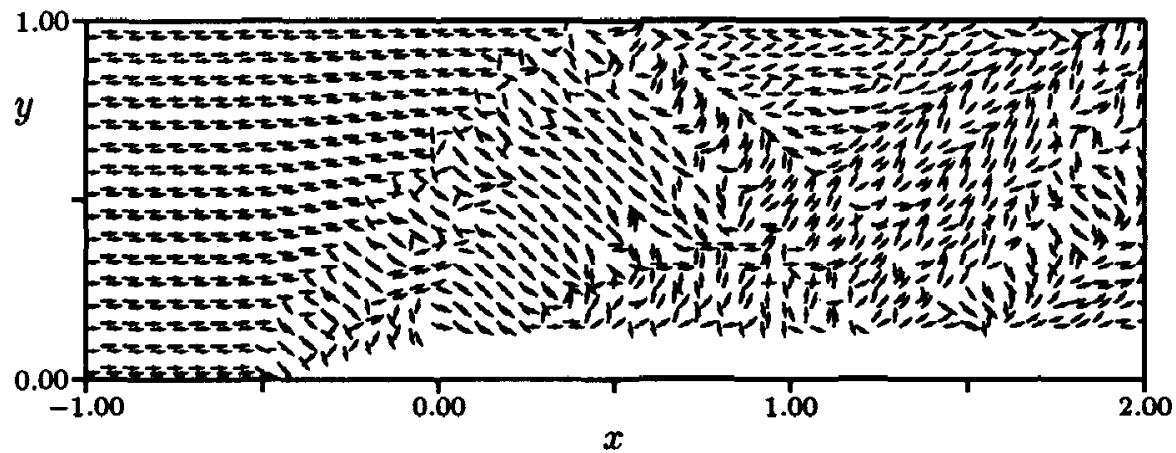

FIG. 14. Upwinding angles for the $15^{\circ}$ wedge flow, $q$-gradient upwinding, $96 \times 32$ cell grid, thinning factor: 2 . 


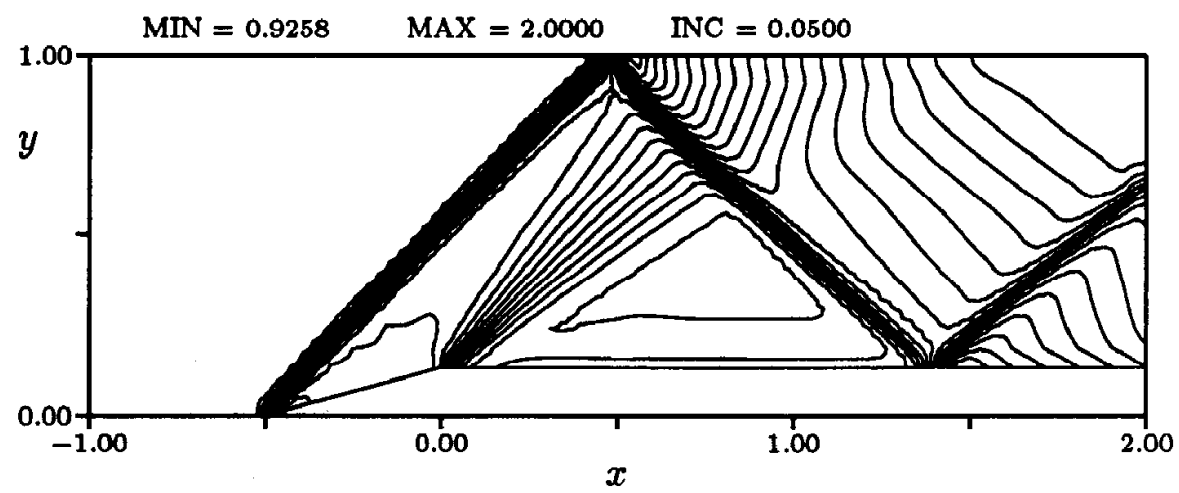

FIG. 15. Mach-number contours for the $15^{\circ}$ wedge flow, $\kappa=\frac{1}{3}$, MUSCL, grid-aligned upwinding, $96 \times 32$ cell grid.

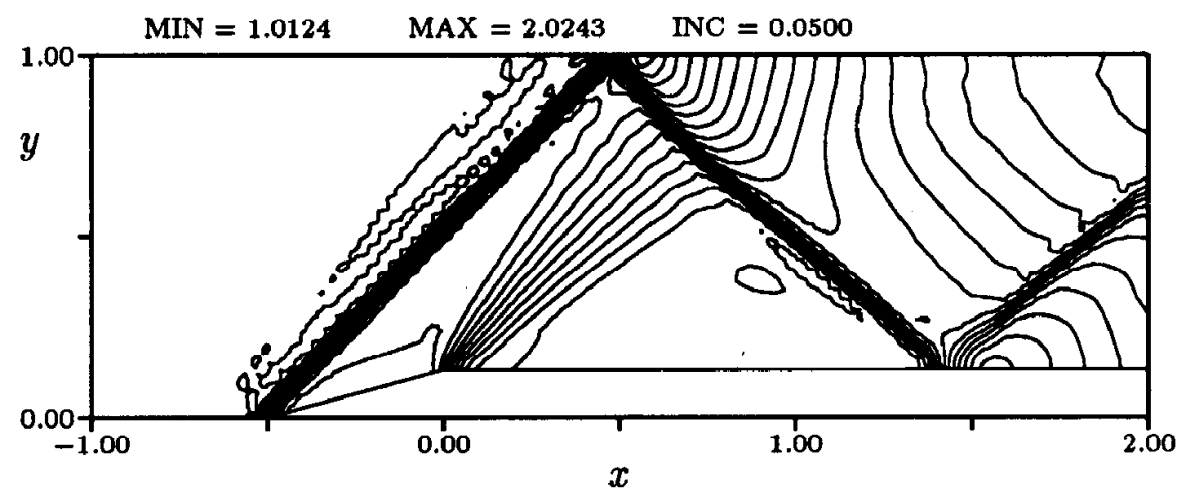

FIG. 16. Mach-number contours for the $15^{\circ}$ wedge flow, first-order $q$-gradient upwinding, Riemann solver for minor fluxes, $96 \times 32$ cell grid.

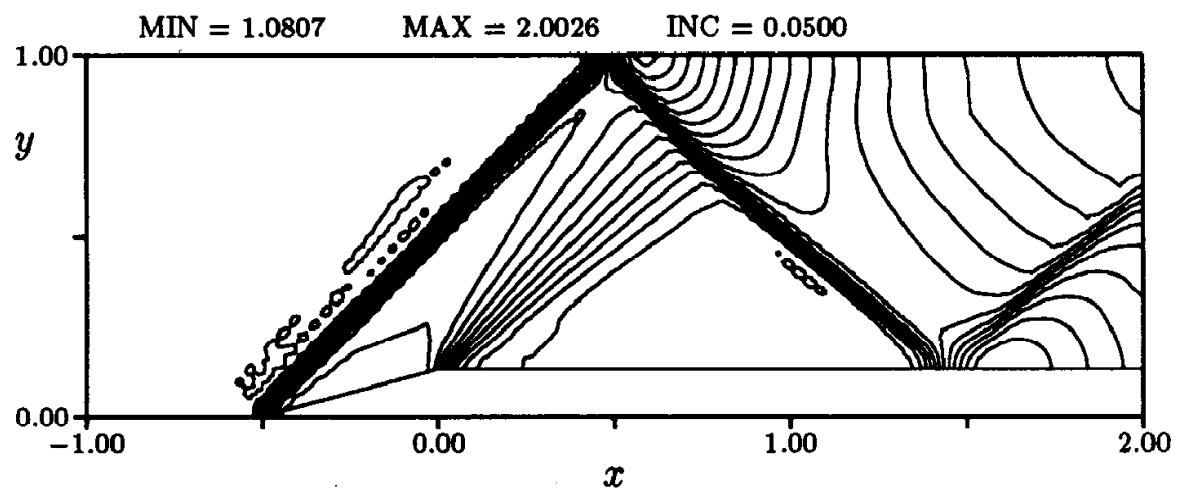

FIG. 17. Mach-number contours for the $15^{\circ}$ wedge flow, limited first-order $q$-gradient upwinding, Riemann solver for minor fluxes, $96 \times 32$ cell grid

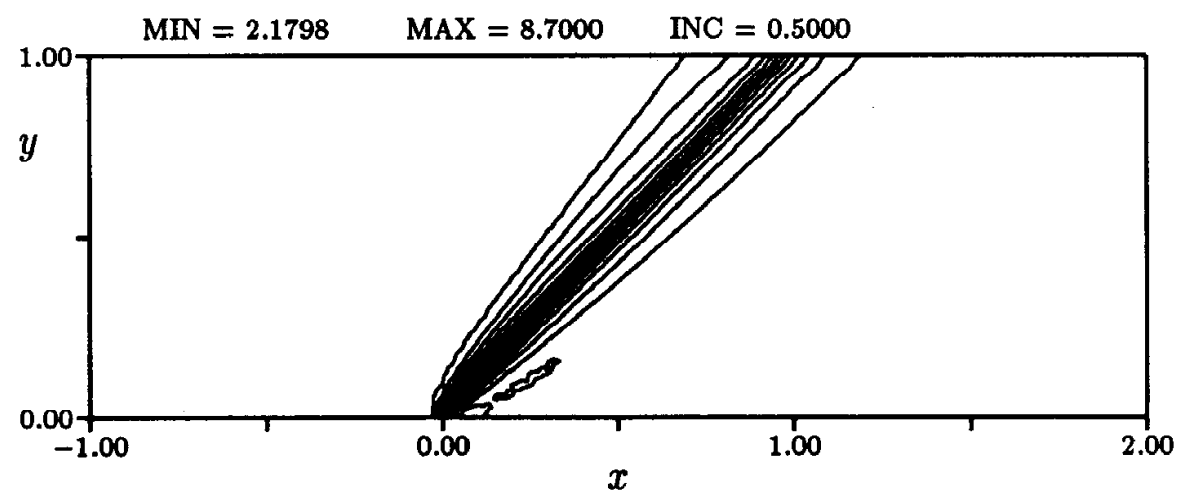

FIG. 18. Mach-number contours for the Mach $8.7 / 2.9, \alpha=45^{\circ}$, shear-wave problem, $\kappa=\frac{1}{3}$ grid-aligned upwinding, $96 \times 32$ cell grid. 


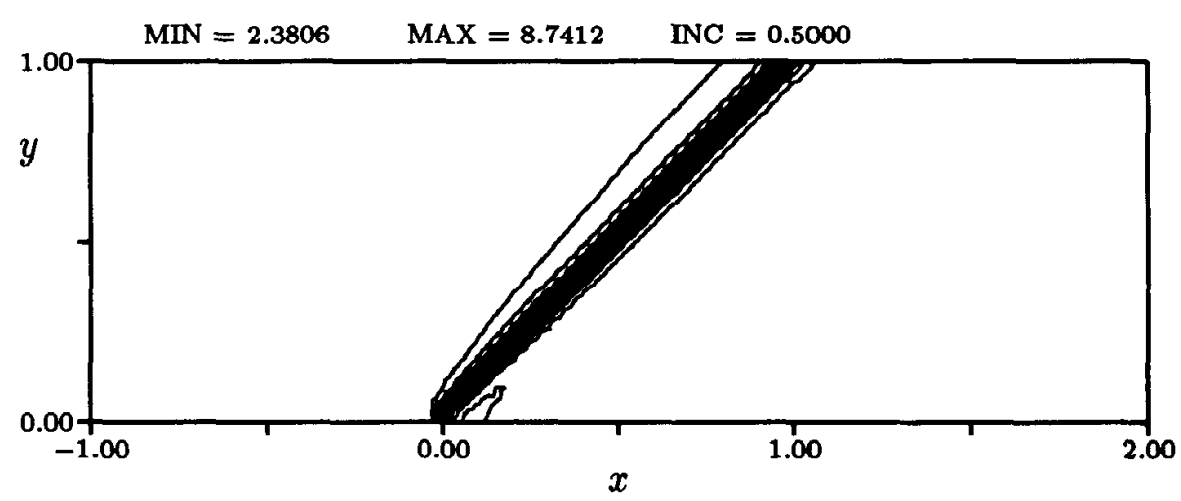

FIG. 19. Mach-number contours for the Mach $8.7 / 2.9, \alpha=45^{\circ}$, shear-wave problem, first-order limited $q$-gradient upwinding, $96 \times 32$ cell grid.

Of the rotated-Riemann-solver schemes, it appears that the $q$-gradient direction is the best choice. It is chosen as the upwinding direction for all the remaining cases.

It is possible that the use of central differencing for the minor fluxes is causing some of the wiggles and destabilizing effects of the present method. In the results shown in Fig. 16, Roe's approximate Riemann solver (the same as used for the dominant fluxes) is used to compute the minor fluxes. While there is a slight reduction in the sharpness of the shocks, the overall quality of the solution has improved, as most of the wiggles are eliminated. For all the remaining cases, the Roe solver is used to compute the minor fluxes.

In Section 2 it was shown that the characteristic interpolation scheme would not be monotone and a method was shown which, through limiting of the upwinding angle, resulted in a monotone scheme. Results for the first-order $q$-gradient scheme with angle limiting are shown in Fig. 17. The results are now essentially non-oscillatory, and the

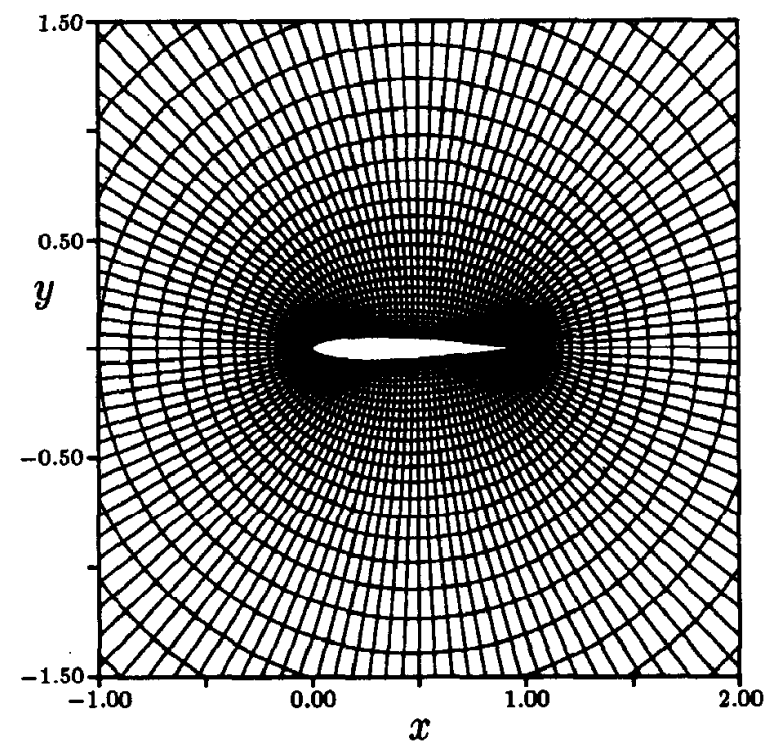

FIG. 20. O-type, $128 \times 32$ cell computational grid for airfoil flow cases. solution is still substantially improved over the first-order grid-aligned results.

Although the directional upwinding shows improved performance in comparison to the grid-aligned method, it comes at a price. The non-linearities introduced by the method cause convergence difficulties. To help remedy this, the upwind angle is recalculated only at specific times: at startup and at intervals of 200 time steps thereafter. In total it is calculated five times.

Shear Wave Oblique to Grid. The next example tests the schemes' ability to resolve a pure shear wave. In this case there is no physical steepening mechanism, as for shock waves, so it better indicates the diffusive properties of a scheme. The flow is inclined at $45^{\circ}$ with respect to the grid, and the flow Mach numbers are 8.7 and 2.9 on the left and right sides of the wave, respectively. Density is unity and pressure is $1 / \gamma$ on both sides of the wave. These conditions are prescribed on the lower boundary, with the wave originating at $x=0$. The grid used is Cartesian.

Results for the $\kappa=\frac{1}{3}$ grid-aligned and the limited $q$-gradient schemes are shown in Figs. 18 and 19. It is seen that the $q$-gradient upwinding better represents the shear wave than the $\kappa=\frac{1}{3}$ grid-aligned method in this case. Part of the reason is the limitation of a cell-face reference frame. The Riemann problems in a frame normal to the cell face will indicate a shock or expansion wave, rather than the pure shear wave that is actually present. The rotated Riemann solver does not have this limitation, so its performance is excellent on this problem.

NACA 0012 Airfoil Case. The previous channel flow cases tested the various schemes on Cartesian or nearCartesian grids. For the airfoil test cases, a conventional O-type grid is used, shown in Fig. 20. The freestream Mach number is 1.20, and the angle of attack is zero. A "bow" shock wave exists in front of the leading edge and "fishtail" shocks emanate from the trailing edge. Results are shown in Fig. 21. The bow and fishtail shock waves are better resolved by the $q$-gradient schemes than by the first-order 

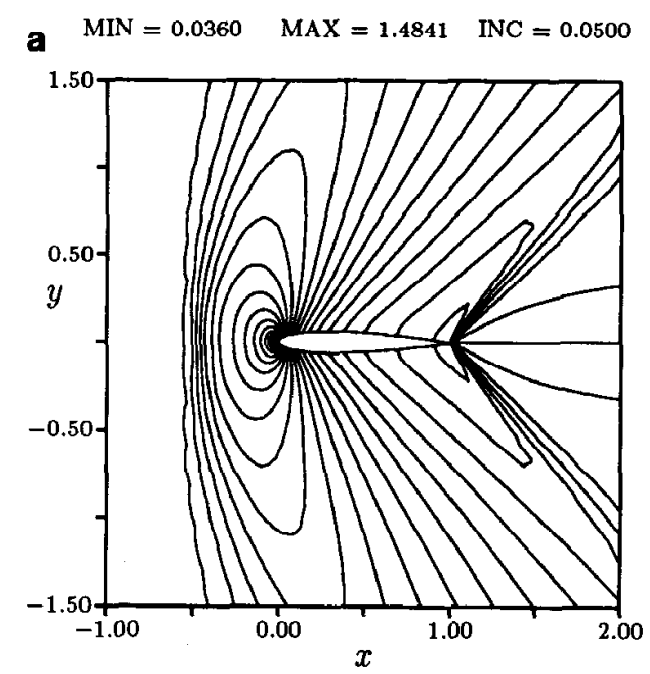

c

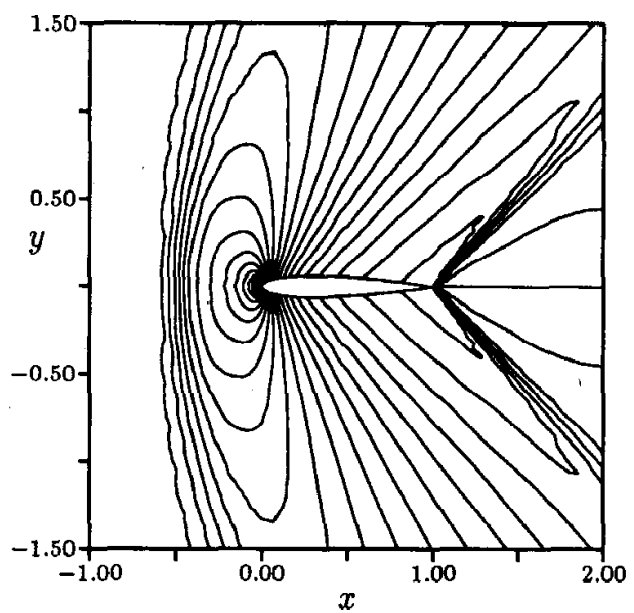

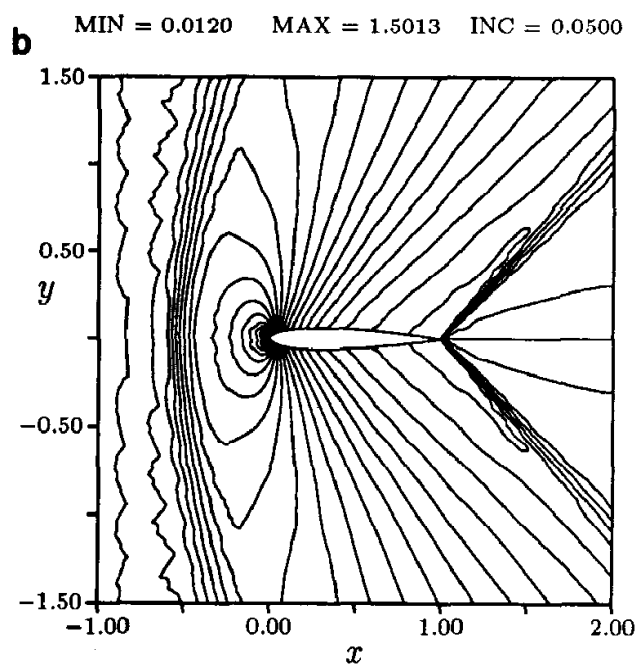

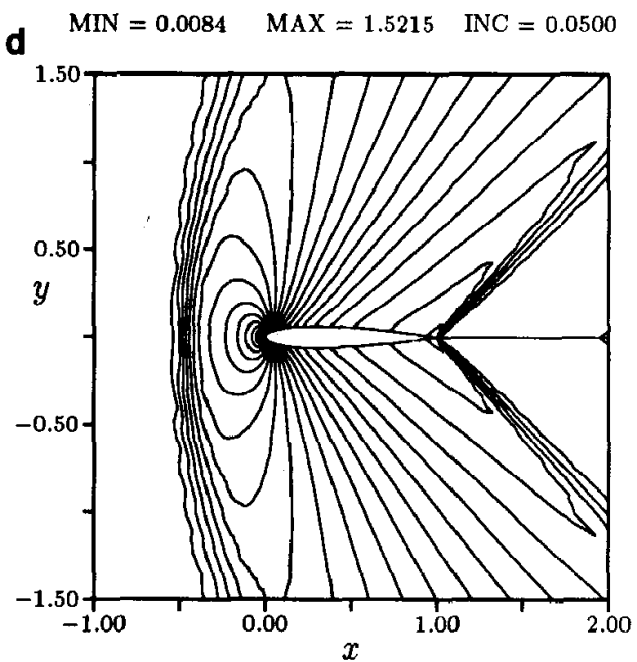

FIG. 21. Mach-number contours for the NACA 0012 airfoil, $M_{\infty}=1.20, \alpha=0.00^{\circ}, 128 \times 32$ cell grid. (a) First-order grid-aligned; (b) first-order full $q$-gradient; (c) first-order limited $q$-gradient; (d) $\kappa=\frac{1}{3}$ grid-aligned.

grid-aligned scheme. Here, the shocks are oblique to the grid, so an improvement is expected. However, the $\kappa=\frac{1}{3}$ grid-aligned scheme still gives the best results. The overshoot ahead of the bow shock in the full $q$-gradient scheme is eliminated by the angle limiting.

\section{CONCLUSIONS AND RECOMMENDATIONS}

In the present research, upwind schemes are formulated for the two-dimensional Euler equations with the direction of upwind differencing derived from strong flow features instead of grid geometry. While multiple strong waves may exist locally in the solution -each with its own optimum upwind direction-a single, dominant, upwinding angle is used, and the resulting improvements are investigated. The gradient of the velocity magnitude, $q$, is chosen as the best direction because it is normal to both shock and shear waves. To obtain input data for the numerical flux function, an interpolation scheme is used to calculate states biased by the chosen upwinding direction. The usual Riemann solver is formulated in the upwinding direction, and an upwindbiased flux is computed in this direction. To complete the flux through the cell face, a Riemann problem is also solved in the direction normal to the $q$-gradient. The upwinding angles are limited to avoid numerical oscillations; the limiting procedure is based on an analysis of scalar convection schemes.

Several test problems are solved, including channel flows and flows about airfoils. The limiting procedure derived for the scalar convection problem is very effective when applied to the rotated Riemann solver, almost completely eliminating overshoots. As with the scalar equation, the rotated Riemann solver generally gives results better than a firstorder grid-aligned method, but slightly inferior to a highorder, grid-aligned, MUSCL scheme. On the other hand, its 
stencil is slightly more compact than that of the MUSCL scheme. The solution to the pure shear-wave problem is the one case where the rotated Riemann solver gives results superior to even that of the best grid-aligned scheme. The rotated Riemann solver finds the reference frame with zero normal velocity, thereby reducing unnecessary dissipation to the minimum possible. This feature would be important in solutions to the Navier-Stokes equations.

Even in cases when it works well, the rotated-Riemannsolver approach still has a great weakness-its expense. The rotated Riemann solver requires a significant amount of additional memory per cell over grid-aligned methods, adds computational effort per iteration and slows convergence. The slower convergence is in part due to the extra nonlinearity of the scheme, embodied in the local rotation angle and limiter.

Despite its problems, the fundamental attraction of the rotated Riemann solver still stands, namely, its respect for the physics of the flow solution. The dramatic improvement in accuracy-compare Figs. 10 and 17-fully justifies the search for grid-independent schemes. Future research may best be concentrated on ways to use the rotated Riemann solver in a selective manner, where it gives superior results, and use high-order grid-aligned upwinding elsewhere. In this manner some of the extra expense may be avoided. Further reduction in expense may be realized if a more efficient interpolation scheme can be developed.

Finally, it must be questioned whether the use of a single, dominant upwind direction is sufficient. In the present research, many inherently one-dimensional ideas are still used even though the upwind direction is based on flow solution features rather than the computational grid. For further improvement to be obtained, a more "systemupwinded" approach may be required, where each state variable may have its own optimum upwind direction, subject to its own limiter.

\section{ACKNOWLEDGMENTS}

This work was funded in part by the McDonnell Aircraft Company under a grant monitored by Dr. August Verhoff, and by the National Science Foundation under Grant EET-8857500, monitored by Dr. George Lea.

\section{REFERENCES}

1. P. L. Roe, Annu. Rev. Fluid Mech. 18, (1986).

2. W. K. Anderson, J. L. Thomas, and B. van Leer, AIAA J. 24 (1985).

3. A. Jameson, Commun. Pure Appl. Math. 28 (1974).

4. A. Verhoff and P. J. O'Neil, Tech. Rep. MCAIR 83-031, McDonnell Aircraft Company, 1983 (unpublished).

5. P. M. Goorjian, "A New Algorithm for the Navier-Stokes Equations Applied to Transonic Flows over Wings," AIAA 8th Computational Fluid Dynamics Conference, 1987.

6. S. Obayashi and P. M. Goorjian, "Improvements and Applications of a Streamwise Upwind Algorithm," AIAA 9th Computational Fluid Dynamics Conference, 1989.

7. G. D. Raithby, Comput. Methods Appl. Mech. 9 (1976).

8. D. Sidilkover, Ph.D. thesis, Weizmann Institute of Science, 1989 (unpublished).

9. S. F. Davis, J. Comput. Phys. 56 (1984).

10. C. Hirsch and C. Lacor, AIAA Paper 89-1958, 1989.

11. K. G. Powell and B. van Leer, AIAA Paper 89-0095, 1989.

12. R. Struijs and H. Deconinck, "A Multidimensional Upwind Scheme for the Euler Equations Using Fluctuation Distribution on a Grid Consisting of Triangles," Eighth GAMM Conference, Delft, 1989.

13. G. Moretti, Comput. Fluids 7 (1979).

14. C. L. Rumsey, B. van Leer, and P. L. Roe, AIAA Paper 91-0239, 1991.

15. A. Dadone and B. Grossman, AIAA Paper 91-0635, 1991.

16. C. Hirsch, C. Lacor, and H. Deconinck, "Convection Algorithm Based on a Diagonalization Procedure for the Multidimensional Euler Equations," AIAA 8th Computational Fluid Dynamics Conference, 1987.

17. P. L. Roe, J. Comput. Phys. 63 (1986).

18. P. De Palma, H. Deconinck, and M. Napolitano, "Cell Vertex Fluctuation Splitting Method Based on Roe's 2-D Wave Models for the 2-D Euler Equations," Von Kármán Institute Internal Report, 1990.

19. K. G. Powell, B. van Leer, and P. L. Roe, "Towards a Geunuinely Multi-Dimensional Upwind Scheme," Computational Fluid Dynamics, Von Kármán Institute for Fluid Dynamics, Lecture Series 1990-04, 1990.

20. P. L. Roe, "Beyond the Riemann Problem," Algorithmic Trends in Computational Fluid Dynamics for the 90s, 1992.

21. P. L. Roe, J. Comput. Phys. 43 (1981).

22. B. van Leer, J. Comput. Phys. 32 (1979).

23. S. K. Godunov, Mat. Sb. 47, 271 (1959).

24. D. Levy, Ph.D. thesis, University of Michigan, 1990 (unpublished).

25. H. W. Liepmann and A. Roshko, Elements of Gasdynamics (Wiley, New York, 1957). 